\title{
Simulation of Cosmic Ray Tau Neutrino Telescope (CRTNT) Experiment
}

\author{
J. L. Liu ${ }^{1}$, S. S. Zhang ${ }^{1}$, Z. Cao ${ }^{1}$, H. H. He ${ }^{1}$, M. A. Huang ${ }^{2}$, \\ T. C. Liu $^{3}$, G. Xiao ${ }^{1}$, M. Zha ${ }^{1}$, B. K. Zhang ${ }^{1}$, Y. X. Bai ${ }^{1}$, \\ Y. Zhang ${ }^{1}$ \\ ${ }^{1}$ Key Laboratory of Astroparticle Physics, Institute of High Energy Physics, \\ Beijing 100049, China \\ 2 Department of Energy and Resources, National United University, 1 Lienda, \\ Miao-Li, 36003, Taiwan \\ ${ }^{3}$ Institute of Physics, National Chiao-Tung University, 1001 Ta-Hsueh Rd, \\ Hsinchu 300 Taiwan \\ E-mail: liujl@ihep.ac.cn
}

\begin{abstract}
A $\tau$ lepton can be produced in a charged current interaction by cosmic ray tau neutrino with material inside a mountain. If it escapes from the mountain, it will decay and initiate a shower in the air, which can be detected by an air shower fluorescence/Cherenkov light detector. Designed according to such a principle, the Cosmic Ray Tau Neutrino Telescope (CRTNT) experiment, located at the foothill of Mt. Balikun in Xinjiang, China, will search for very high-energy cosmic $\tau$ neutrinos from energetic astrophysical sources by detecting those showers. This paper describes a Monte Carlo simulation for a detection of $\tau$ events by the CRTNT experiment. Ultra-high-energy cosmic ray events are also simulated to estimate the potential contamination. With the CRTNT experiment composed of four detector stations, each covering $64^{\circ} \times 14^{\circ}$ field of view, the expected event rates are 28.6, 21.9 and 4.7 per year assuming AGN neutrino flux according to Semikoz et. al. 2004, MPR AGN jet model and SDSS AGN core model, respectively. Null detection of such $\tau$ event by the CRTNT experiment in one year could set $90 \%$ C.L. upper limit at $19.9\left(\mathrm{eV} \cdot \mathrm{cm}^{-2} \cdot \mathrm{s}^{-1} \cdot \mathrm{sr}^{-1}\right)$ for $E^{-2}$ neutrino spectrum.
\end{abstract}

PACS numbers: 96.85.Ry, 96.40.Pq, 98.70.Sa, 95.55.Vj

Submitted to: J. Phys. G: Nucl. Phys. 


\section{Introduction}

The origin of cosmic rays (CR) above $10^{15} \mathrm{eV}(1 \mathrm{PeV})$ has been a long-standing problem. Observation of neutral particles, which can be directly traced back to the sources, is a unique way to search for point sources of CRs under $10^{19} \mathrm{eV}$. There are many energetic astrophysical sources such as Gamma Ray Bursts (GRB) or Active Galactic Nuclei (AGN). Those objects could accelerate particles to ultra-high energy and produce CRs, gamma rays, and neutrinos [1, 2]. For photons at $10^{14} \mathrm{eV}$ to $10^{16}$ $\mathrm{eV}$, their attenuation length for interaction with cosmological microwave background photons are approximately $10 \mathrm{kpc}$, roughly the distance from the solar system to the galactic center. Neutrinos are the only choice for exploring remote CR sources. Observation of ultra-high-energy neutrinos is not only an important proof of their existence but also an orthogonal tool, besides conventional astronomical tools such as optical and radio observation, for studying GRBs or AGNs. However, because of very weak interaction, neutrino detection requires a huge volume of detector medium. Conventional way of neutrino detection is to build or locate a huge volume of target and bury detectors inside so that charged particles as products of interaction between incident neutrinos and target material can be detected. As an example, the IceCube experiment, located near the geographic South Pole in Antarctica, uses natural ice approximately $2 \mathrm{~km}$ under the surface as the target and strings of UV sensitive optical modules buried in the ice to detect Cherenkov light generated by muons and electrons that are produced in interaction of incident neutrinos 3 . Working in the similar principle, the Antares experiment uses natural water in Mediterranean sea instead of ice and sinks strings of similar optical modules down to the sea bed to detect Cherenkov photons 4. The main advantage of underwater/ice neutrino detectors is in the ability to collect muons from large distances since muon range is much longer compared to electron or tau range. For electrons generated by e-neutrino, cascade in the sensitive area of the detector can be reconstructed. For $\tau$ neutrinos in certain energy range, both interaction vertex where $\tau$ lepton is produced and $\tau$ decay vertex might be contained in the sensitive area so that yields a clear signature of "double bang". Therefore, the detector is sensitive to all species of incident neutrinos with somewhat clear signature for different species. Due to a limited attenuation length of Cherenkov light, spacing between optical modules must be sufficiently small in order to collect sufficient Cherenkov photons that enables reasonable reconstruction of tracks or showers inside the detector. This is a disadvantage of this type of detector in terms of costs of construction. It is substantial in the case of neutrino observation with very low statistics, e.g. typically tens of events per year for a scale of experiments such as IceCube. To seek some more economic ways to build larger detectors for statistically significant measurements is in fact essential. Sometimes, one has to sacrifice some performance of the detector to fulfill the goal. One of many ways to explore economic detection of ultra-high-energy neutrinos is to separate the detector volume from the target volume in which the incident neutrino interacts. By doing so, the target volume can be as large as a piece of mountain or even a part of the earth shell without putting any detector inside, and the detector volume can be as large as the nearby atmosphere, such as that for the HiRes experiment [5] and the Pierre Auger Observatory (PAO) experiment [], both are primarily designed for detecting cosmic rays above $1 \mathrm{EeV}$. An obvious disadvantage is that the interaction vertex is no longer in the scope of the detector. A potential difficulty might be that the observation could be contaminated by the cosmic ray background. There exists a 
solution to avoid the cosmic ray background by slightly reduce the detector volume with a more dedicated design of configuration that uses the fluorescence/Cherenkov telescopes, such as HiRes/Auger telescopes, to watch a volume of a few kilometers behind a mountain and keep the field of view of those telescopes in the shadow of the mountain. The mountain plays not only a role as a huge target, but also a screen of cosmic rays coming into the field of view of the detector. By mainly collecting Cherenkov photons, instead of fluorescence photons, generated in air showers, such an array of telescopes can work at energies as low as $1 \mathrm{PeV}$, the same energy range as the IceCube experiment. This technique is reasonably inexpensive and the detector aperture can be easily increased in scale to compensate for the disadvantage of this technique only effectively detecting tau neutrinos. In the rest of this paper, we are discussing detailed physics perspectives with one of specific configuration of this type of detector.

Before going to more details, let us briefly review the principle of the detection method. Incident neutrinos convert into electrons, muons and taus in a mountain through the charged current interaction depending upon their flavor. Electrons will shower quickly inside the target material. Muons travel very long distance before decaying, therefore, they are not easy to be detected using this method. $\tau$ leptons produced inside the mountain, that have sufficiently long life-time to escape from the mountain, decay and induce showers in the air. Conventional air shower detector, such as fluorescence/Cherenkov telescopes, can be used then to detect them. The Cosmic Ray Tau Neutrino Telescope (CRTNT) experiment is designed to detect those neutrino-induced air showers [7]. To find a suitable site for the experiment is not trivial because it must be sufficiently dry year round and the mountain must be sufficiently steep so that there is enough space for showers well developed to be detectable. Strongly depending upon the specific thickness of the mountain and terrain of the site, the CRTNT experiment needs to be reevaluated for its feasibility with very much updated AGN neutrino models and simulation tools. Since publication of the reference [7. which addressed basic principle of the detection, there are many progresses in AMANDAII data analysis, neutrino models and simulation techniques. The purpose of this paper is to address them in somewhat details.

The rest of the paper is organized as follows. The configuration of the CRTNT detector is described in Section 2. Section 3 details the procedure of $\tau$ neutrino converting into $\tau$ lepton in the mountain and detecting of air shower initiated by the $\tau$ lepton. In Sections 4, 5 and 6, the event rate and sensitivity of the CRTNT detector are estimated. We summarize all results for this study and compare with previous works in the last section.

\section{The CRTNT Project}

The proposed CRTNT project, which currently has two telescopes as a prototype running at Tibet site 8 and detecting cosmic ray showers that observed by the ARGOYBJ experiment [8], uses fluorescence/Cherenkov light telescopes to detect air showers induced by tau neutrinos. A candidate site is at the foothill of Mt. Balikun, about $130 \mathrm{~km}$ north of Hami, Xinjiang province, China. The contour map of the mountain is shown in figure1. The mountain range runs in east-west direction and the northern side is quite steep. The height of the mountain stays at about $4000 \mathrm{~m}$ a.s.l. for more than $30 \mathrm{~km}$ in east-west direction. At the foothill, Balikun valley stretches hundreds of kilometers at a height of approximately $1500 \mathrm{~m}$ a.s.l. The total precipitation is less 
than $200 \mathrm{~mm}$ per year. The Balikun site provides an excellent convertor for mountainpassing neutrino events and suitable weather condition for observing the resultant air showers with fluorescence/Cherenkov light telescopes. Preliminarily selected sites for four CRTNT arrays, denoted as FD1, FD2, FD3 and FD4, are located in the northern valley within $30 \mathrm{~km}$ from Balikun city. All sites are convenient in terms of accessibility of power supply and major highways. The ideal orientation of field-of-view (FOV) of the detector enables a decent observation of the galactic center, which is considered as the most favorable neutrino source in our galaxy, with a considerable large exposure. An optimized configuration of the detector array is shown in figure1.

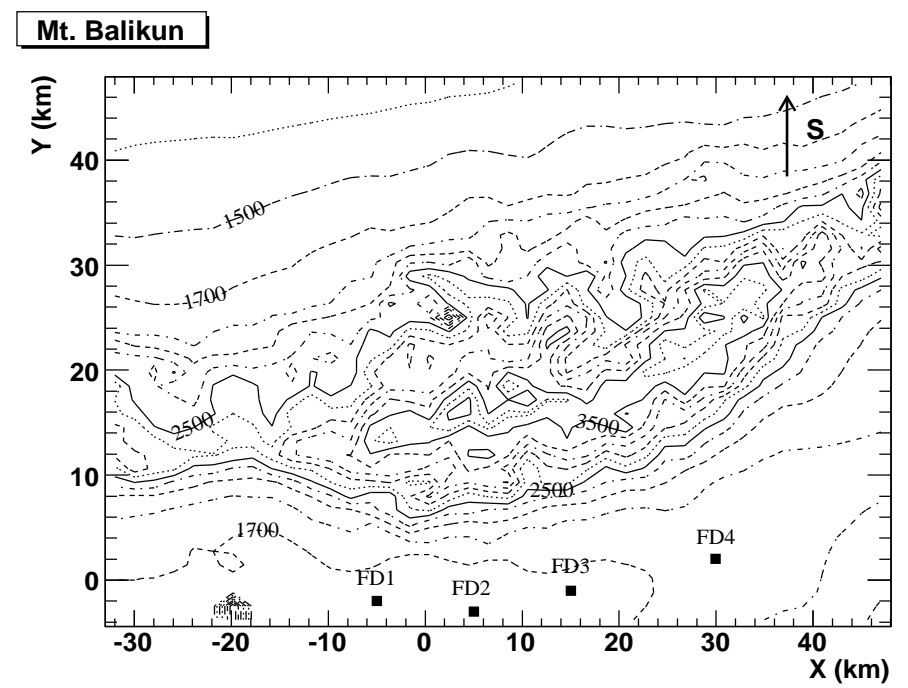

Figure 1. Contour map of Mt. Balikun; the numbers represent the altitudes in meter. Solid rectangles are the location of four CRTNT telescope sites.

At each site, four telescopes observe a FOV of $64^{\circ}$ in azimuth angle and $14^{\circ}$ in elevation angle. Each telescope has a mirror of $5 \mathrm{~m}^{2}$ with a reflectivity of $82 \%$. A focal plane camera consists of $16 \times 16$ photomultiplier tubes (PMTs). Each PMT has a hexagonal photo-cathode of $44 \mathrm{~mm}$ from side to side with a FOV of approximately $1^{\circ} \times 1^{\circ}$. Signals from tubes are read out by $50 \mathrm{MHz}$ flash ADCs to record their complete waveforms. An algorithm for finding pulse area is used to determine how many photons are detected by each channel.

A successful triggered event must pass three levels of trigger criteria. The firstlevel trigger is formed in every single channel, referred to as tube trigger. It requires the signal-to-noise ratio to be greater than $4 \sigma$, where $\sigma$ is the standard deviation of the total photon-electron noise within a running window of $640 \mathrm{~ns}$. The second-level trigger is formed with coincidence among channels that forms certain patterns in the camera, on the basis of a pattern recognition algorithm, referred to as the telescope trigger. "Track-type" pattern requires at least six triggered pixels forming a straight line corresponding to a shower passing through the FOV. "Circular-type" pattern requires seven triggered pixels forming a solid circle corresponding to a Cherenkov image of a shower pointing toward the telescope. The patterns are searched within a $6 \times 6$ box running over the entire camera. The third-level trigger for an event requires at least one telescope to be triggered. 


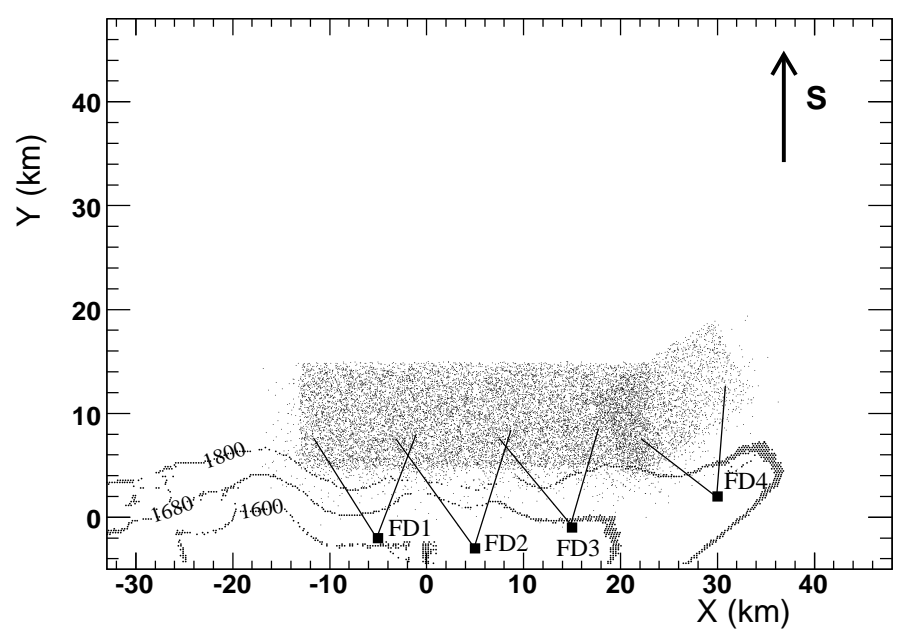

Figure 2. Starting points of showers initiated by $\tau$ leptons. Solid rectangles represent four potential CRTNT sites and touched lines indicate the FOV of telescopes on each site. The dotted curves show the mountain profile near the four sites.

\section{Monte Carlo Simulations}

Simulation of a $\tau$ neutrino event is divided into the following three stages: 1) the $\tau$ neutrino interacts inside the mountain; 2) the $\tau$ lepton decays and initiates an air shower; and 3) photons are generated from the shower and detected by the CRTNT detector. To estimate the background for the neutrino detection, CR showers flying over the mountain are also generated. Both the fluxes of neutrinos and CRs are assumed to be isotropic and uniform in the FOV of the detector.

\subsection{Tau neutrino interaction}

Primary neutrinos ranging from $1 \mathrm{PeV}$ to $6 \mathrm{EeV}$ are sampled according to models that predicts fluxes from active galactic nuclei (AGN) 9, 10, 11, 12. Incident neutrinos are uniformly distributed from $73^{\circ}$ to $101^{\circ}$ in zenith angle and from $0^{\circ}$ to $180^{\circ}$ in azimuth angle (the west is defined as $0^{\circ}$ in azimuth). All neutrinos enter the mountain from the southern side. A three-dimensional global coordinate system is employed to describe incident directions, interaction positions of $\tau$ neutrinos, and mountain profile, which are modeled by a digital topological map. A one-dimensional coordinate system along the trajectory is defined to describe all the three stages. If an incident $\nu_{\tau}$ interacts inside the mountain, the energy and momentum of the produced $\tau$ are traced until it decays. The energy loss and the decay position of $\tau$ are simulated. Regeneration of $\nu_{\tau}$ is taken into account, i.e. if the $\tau$ decays inside the mountain, decay product $\nu_{\tau}$ will be traced using the same procedure again.

SHINIE (Simulation of High-energy Neutrinos Interacting with the Earth), a Monte-Carlo simulation package, is used in description of neutrino interaction in our simulation. In this package, the inelasticity $y$ and the differential cross-section $d \sigma / d y$ are calculated separately, using the latest parton distribution function CTEQ6 [13]. 
The newly calculated cross section below $1 \mathrm{EeV}$ is about $7 \%$ less than the result in the package LEPTO 14. Tau/muon lepton energy loss is also calculated in details. The detailed description can be found in [15] and references therein.

\subsection{Tau decay and air shower initiation}

Once a $\tau$ lepton escapes from the mountain and decays in the air, energies of daughter particles are simulated using TAUOLA [16] for all decay channels. Electrons and hadrons (the branch ratio of $83 \%$ ) will initiate air showers. The air shower carries approximately one half of the $\tau$ energy. The starting point of shower along the particle trajectory is calculated according to the random sampling of $\tau$ decay length, while the shower direction is the same as that of the primary $\nu_{\tau}$. The projection of all the starting points are shown in figure2. If decays occur behind the detector, the shower cannot be detected and are ignored in the simulation.

Corsika (version 6.611) [17] is employed to generate air showers in the space between shower initiating points and the CRTNT detectors. A pre-simulated shower library is established at 33 selected energies distributed between $0.1 \mathrm{PeV}$ and $0.6 \mathrm{EeV}$. At each energy, 100 hadronic (pion as the primary particle) and 100 electromagnetic (electron as the primary particle) showers are simulated. The longitudinal profiles of showers, i.e. number of charged particles as a function of slant depth at every $5 \mathrm{~g} / \mathrm{cm}^{2}$ in the air, are recorded in the library. The depth of air is calculated according to the US standard atmosphere model (1976) 18 . The earth curvature is taken into account.

A shower profile is randomly selected from the library according to the particle identification at the closest energy and the number of charged particles in a shower is scaled up or down to represent the shower to be simulated.

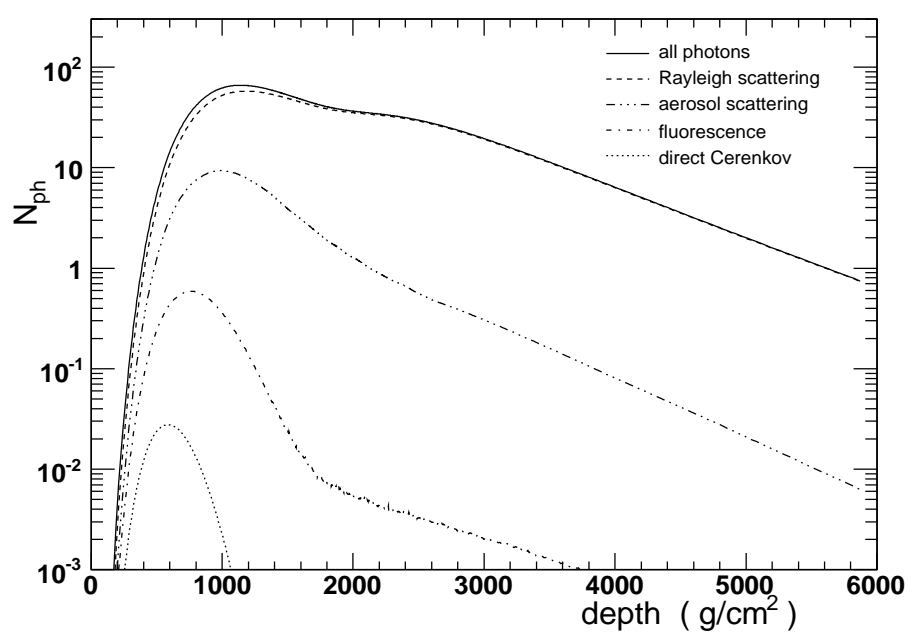

Figure 3. Profiles of all photons seen by the detector along the shower longitudinal development of a typical event. The solid curve is the sum of all photons. The dashed curve is Cherenkov photons scattered by atmospheric molecules (Rayleigh scattering). The double-dot-dashed curve is Cherenkov photons scattered by aerosols. The dot-dashed curve is fluorescence photons. The dotted curve represents direct Cherenkov components. 


\subsection{Photon production and the CRTNT detector simulation}

Ultraviolet fluorescence light is generated as charged shower particles passing through the atmosphere. Laterally, fluorescence photons are assumed to follow a distribution of electrons described by Nishimura-Kamata-Greisen (NKG) formalism[19]

$$
\rho(r)=\frac{N}{\left(r_{0}\right)^{2}} f\left(s, \frac{r}{r_{0}}\right) .
$$

where $r_{0}$ is the Moliere unit, and $s$ is the age of the shower. The normalized function $f$ reads as

$$
f\left(s, \frac{r}{r_{0}}\right)=\left(\frac{r}{r_{0}}\right)^{s-2}\left(1+\frac{r}{r_{0}}\right)^{s-4.5} \Gamma(4.5-s) /[2 \pi \Gamma(s) \Gamma(4.5-2 s)] .
$$

Cherenkov photons are also generated by charged particles if the particle energy is higher than the threshold energy. Photons scattered by the atmospheric molecules and aerosols are distributed in all directions according to corresponding phase functions. A standard desert aerosol model 20, with a scale height of $1 \mathrm{~km}$ and a horizontal attenuation length of $25 \mathrm{~km}$ is assumed in the simulation. A ray-tracing procedure is carried out to trace each photon to the photo-cathodes of PMTs once photons are generated. All detector responses are considered in the ray-tracing procedure. A detailed description of the ray-tracing procedure can be found elsewhere 21] and references therein. In figure3, profiles of all kinds of photons that collected by the CRTNT detector are plotted as functions of slant atmospheric depth along the shower longitudinal development for a typical simulated shower.

All photons collected by one PMT form a complete waveform according to their arrival time. Night sky background (NSB) photons with a flux of 40 photons per microsecond per square meter of the light collector are randomly added to the waveform. The electronic noise with a mean of 1.2 FADC counts per $20 \mathrm{~ns}$ is also added to every channel. Triggering algorithm at three levels as described in Section 2 is implemented in the simulation. In figure4, an example of a neutrino-induced shower event detected by CRTNT is plotted.

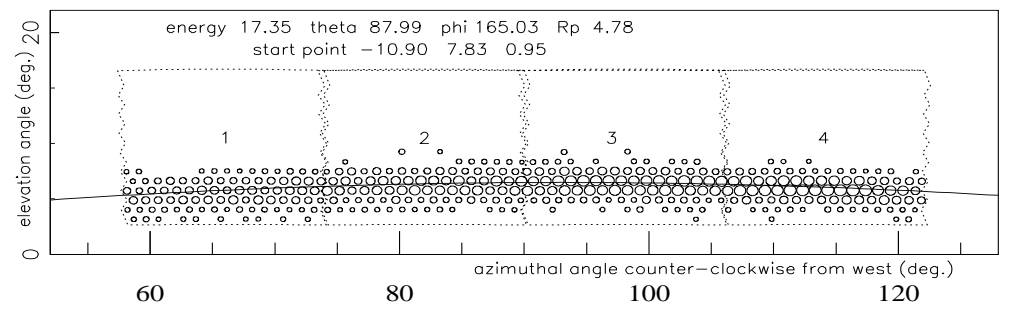

Figure 4. A typical horizontal air shower seen by the CRTNT detector. Each square marked with a number shows the FOV of each telescope. Circles represent triggered tubes and the size of each circle is proportional to logarithm of the number of photons. The solid line represents a plane containing shower axis and the detector. Energy (in PeV), zenith and azimuth angle (in degree), impact parameter and coordinates of shower starting point (in $\mathrm{km}$ ) are displayed. 


\subsection{Cosmic ray background simulation}

As a background, CR showers are simulated with core location uniformly distributed in an area of $32 \mathrm{~km} \times 10 \mathrm{~km}$ between the mountain and detectors. CR shower energies are randomly selected from a spectrum of $E^{-3}$ between $3 \mathrm{PeV}$ to $1 \mathrm{EeV}$. All showers are between $70^{\circ}$ and $75^{\circ}$ in zenith angle and between $0^{\circ}$ and $180^{\circ}$ in azimuth angle. Cosmic rays from smaller zenith angles $\left(\theta<70^{\circ}\right)$ can be rejected according to their reconstructed geometry without any ambiguity. CRs from a larger zenith angles $\left(\theta>75^{\circ}\right)$ are screened by the mountain.

Using Corsika (version 6.611), a shower library similar as that in neutrino simulation at 26 selected energies distributed from $3 \mathrm{PeV}$ to $1 \mathrm{EeV}$ and zenith angle at 75 degrees is established. At each energy, 100 hadronic showers with proton as primary particles are produced.

In the simulation, cosmic ray showers are randomly selected from the shower library. The slant atmospheric depth with an earth curvature correction, photon production, light propagation and trigger algorithm are the same as those used in the neutrino simulation. A uniform mountain profile with a height of $2.5 \mathrm{~km}$ is assumed to be $8 \mathrm{~km}$ away from the detectors serving as a screen.

\section{Estimation of event rate}

Using the algorithm described in Section 3 , a $\nu_{\tau}$-to-shower conversion efficiency of $1.92 \times 10^{-4}$ is yielded. An average trigger efficiency of showers induced by the products of $\tau$ decay is found to be approximately $21.8 \%$. The input and observed event distribution is shown in figure5. By using the AGN neutrino flux proposed by 9], the event rate is 35.7 per year assuming a duty cycle of $15 \%$ for the CRTNT detector. Although this AGN flux model was ruled out by the AMANDA experiment 22 we still use it for comparison with our previous study [7, where the same model was used.

We also estimate the event rates of other two AGN models. The $\nu_{\tau}$ to $\tau$ conversion efficiency of MPR AGN jet model[10] is $2.14 \times 10^{-4}$. The triggering efficiency is $23.5 \%$. The expected event rate is 27.3 per year. For SDSS AGN core model 11, 12, the $\nu_{\tau}$ to $\tau$ conversion and triggering efficiency are $7.25 \times 10^{-5}$ and $12.5 \%$, respectively. The expected event rate is 5.9 per year for this model.

Event rate of CR showers is estimated using average flux $J(E)=2 \times 10^{24} \times$ $E^{3}\left(\mathrm{eV}^{2} \cdot \mathrm{m}^{-2} \cdot \mathrm{s}^{-1} \cdot s r^{-1}\right)$ [23]. The trigger efficiency is found to be about $0.29 \%$ for CR showers. The input and observed spectra are shown in figure6. In the simulation corresponding to an exposure of more than two years, $93407 \mathrm{CR}$ events are collected, i.e. 38300.7 events will be detected per year.

\section{Event selection}

Neutrinos and CRs produce showers with distinct characteristics because they come from different directions and develop in different depths of the atmosphere. Without detailed shower reconstruction, a simple algorithm can identify neutrino events from CR background events by sorting potential neutrino events into the following five types.

(i) Up-going event

A shower detector plane (SDP) is defined as a plane that contains the shower 


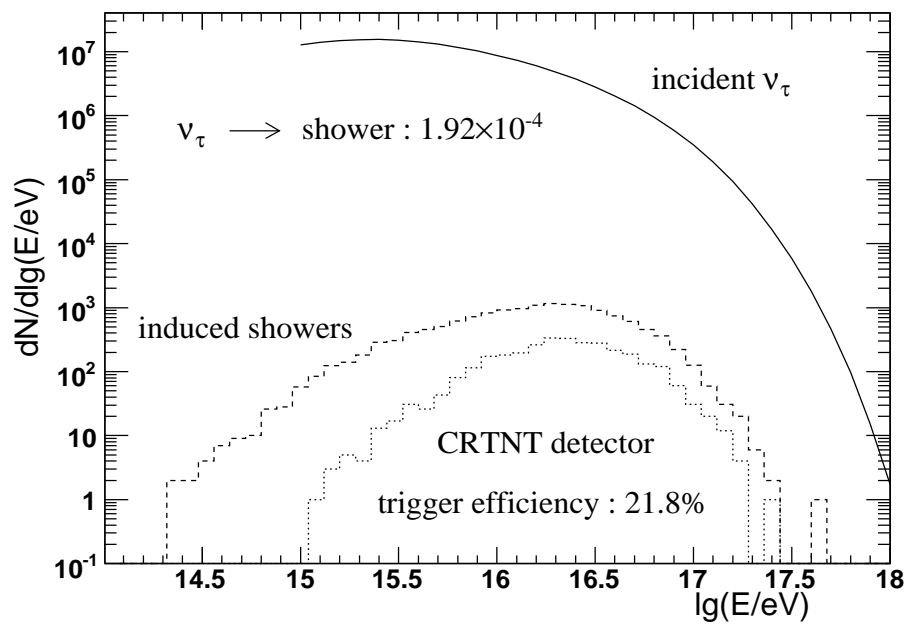

Figure 5. Input and observed event distribution using CRTNT array in Mt. Balikun. The solid curve represents the incident neutrino spectrum, the dashed curve is the induced shower energy distribution, and the dotted curve shows the triggered events energy distribution.

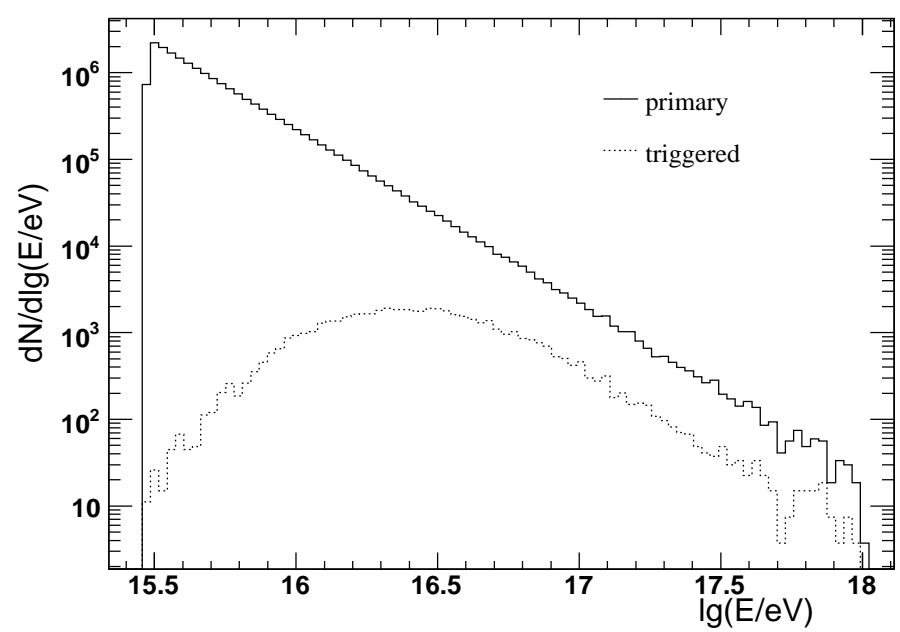

Figure 6. Energy distribution of primary CRs (solid curve) and triggered events (dashed curve). 
axis and the detector. An apparent up-going event, caused by neutrinos, can be identified by finding the elevation angles of triggered tubes along the SDP increasing with time. Only by checking on the shower flying direction, a cut criterion in time difference between the highest and the lowest tubes, denoted as $d t$, will pick out the majority of neutrino events $(41.5 \%)$. The $d t$ distributions for apparent up-going neutrino $(d t>0)$ and all CR showers are shown in figure7(a). In order to avoid cosmic ray showers that apparently go upwards, a cut at $200 \mathrm{~ns}$ is chosen.

(ii) Horizontal event

An event whose SDP normal vector lies within a cone of 5 degrees from zenith is called a horizontal event. Such events might not be picked up as neutrino events by timing (i). The space angle between SDP normal vector and the zenith, denoted as $\psi$, distributes as in figure7(b). After the above step/s (the same in the following), approximately $7.9 \%$ of neutrino events are of this type. No cosmic rays belong to this category.

(iii) Neutrino induced Cherenkov event

Head-on showers generate Cherenkov images concentrating in an ellipse-like pattern. Because all photons arrive to the detector almost at the same time, timing criterion does not work for this type of events. However, a neutrino induced Cherenkov event must have the elevation angle of the center of image (COM), defined as a mean position weighted by triggered PMT signals, lower than $11^{\circ}$, because they come out from the mountain. CR events from such low directions start at very far away and are blocked by mountains. Only small portion of them which just fly over the tip of the mountain may generate similar patterns, but the COMs must be at high positions. Figure7(c) shows distributions of elevations of the COMs for different showers. A cut at $11^{\circ}$ makes a clear separation between them. Approximately $17.7 \%$ of neutrino events belongs to this category.

(iv) Very long track event

Once an energetic shower starts at outside the FOV of the detectors, their shower tracks could be observed by multiple mirrors at one site as a characteristic. If it is induced by a mountain-passing neutrino, it is once again characterized by its low elevation of the COM. Therefore, cutting on both COM elevation and number of rows of triggered tubes in a shower as shown in figure7(d), i.e. the COM elevation must be lower than 10deg and number of rows must be less than 13 , neutrino events will be picked out. Only $7.2 \%$ events among all neutrino events belong to this category.

(v) Back-to-mountain event

There must be some events with very clear characteristics that the first triggered tube located in the central area of the FOV of a telescope, thus strongly indicate that $\tau$ leptons come out from the mountain and initiate a shower start from where the tube points at. Without many efforts, those neutrino events should be picked out if shower images start from points that are certainly not associated with any edges of the FOV. CR shower images must engaged with the edges because the FOV is screened by the mountain. As a quantitative criterion, 4 deg along SDP between the start point of an image to one of the edges is applied to select this type of events. This takes the reconstruction error of SDP into account. However, only $5.8 \%$ of neutrino events are falling in this category. 

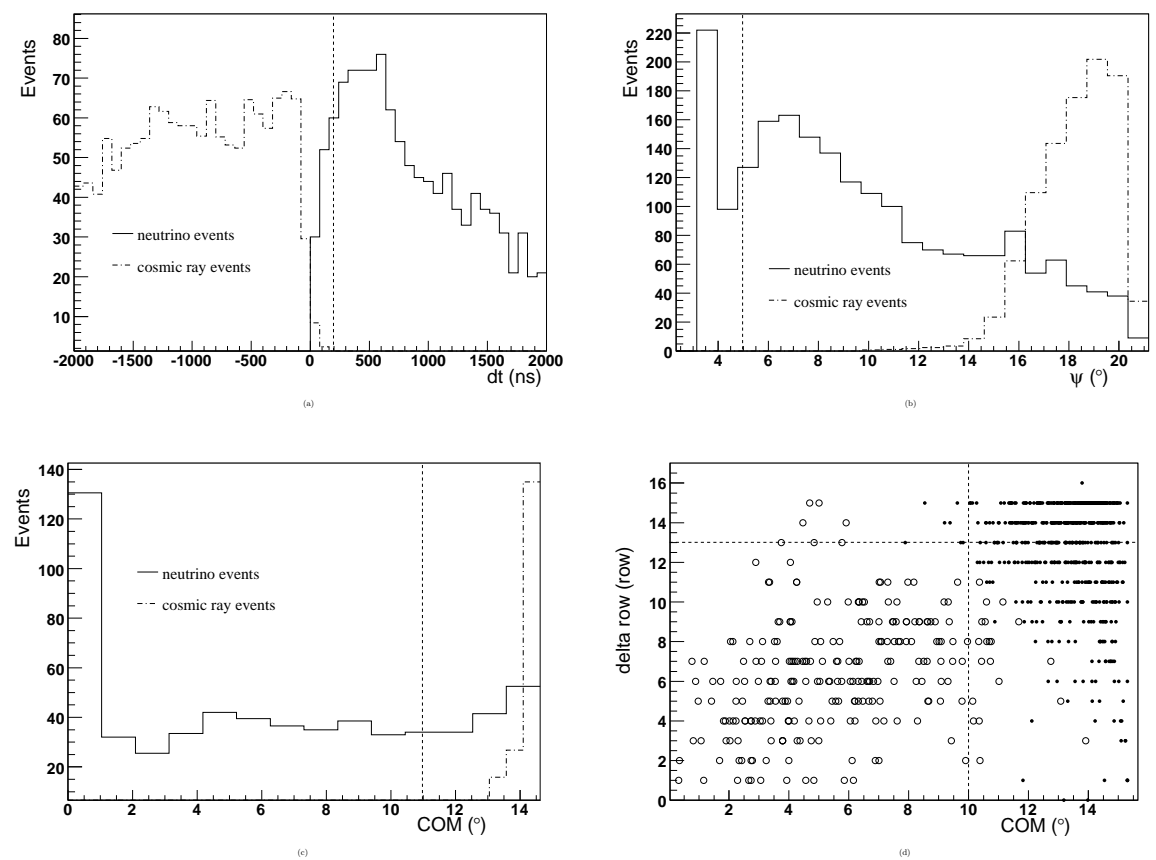

Figure 7. Parameters distribution for neutrino induced events (solid curves in figure (a), (b) and (c), open circle in figure (d)) and cosmic ray induced events (dash-dotted curves in figure (a), (b) and (c), solid circle in figure (d)). The dashed lines represent the cut criteria. (a) Average flying time for a shower over the FOV of CRTNT detector. (b) The distribution of the space angle between SDP normal vector and zenith. (c) COM elevation distribution for the cherenkov events. (d) Number of rows of shower images versus elevation of the center of image for very long track events. (see text for more details.)

Summing them up, $80.1 \%$ of neutrino showers can be identified. The event rate for AGN(Semikoz et al., 2004) reduces to 28.6 per year. The event rates for MPR AGN jet model and SDSS AGN core model are 21.9 and 4.7 per year, respectively.

To estimate how many CR showers are mis-identified as neutrino events, we apply the proposed event selection algorithm to $93407 \mathrm{CR}$ events, and only one of them is selected as a neutrino event. This yields an average CR background of 0.4 event per year for the neutrino detection.

\section{Sensitivity}

We estimate the sensitivity of CRTNT project using modified Feldman-Cousins method 24. The survival probability of triggered neutrino events, defined as the number of identified neutrino events out of the total, is used as a reference parameter in the optimization procedure. By tuning the criteria, the survival probability changes from $67 \%$ to $92 \%$ as listed in Table 1 . The minimum upper limit goes to $19.9\left(\mathrm{eV} \cdot \mathrm{cm}^{-2} \cdot \mathrm{s}^{-1} \cdot \mathrm{sr}^{-1}\right)$ with a hypothetical neutrino flux $\Phi_{s}=E^{2} \cdot J(E)=$ $10^{2}\left(\mathrm{eV} \cdot \mathrm{cm}^{-2} \cdot \mathrm{s}^{-1} \cdot \mathrm{sr}^{-1}\right)$ with energies from $10^{15} \mathrm{eV}$ to $10^{18} \mathrm{eV}$.

As the result, if no excess is observed in one year, an upper limit of $19.9(\mathrm{eV}$. 
Table 1. Optimization procedure of neutrino event selection.

\begin{tabular}{ccccc}
\hline$R(\%)$ & $n_{s}$ & $\left\langle n_{b}\right\rangle$ & $\overline{\mu_{90}}$ & upper limit \\
\hline 67.4 & 11.73 & 0.00 & 2.44 & 20.8 \\
80.1 & 13.94 & 0.41 & 2.78 & 19.9 \\
85.3 & 14.84 & 0.80 & 3.03 & 20.4 \\
92.0 & 16.08 & 1.23 & 3.46 & 21.5 \\
\hline
\end{tabular}

$R$ is the neutrino event survival probability. $n_{s}$ represents the expected number of events per year from the model of $\Phi_{s}$ after the event selection. $\left\langle n_{b}\right\rangle$ is the corresponding number of background cosmic ray events per year. $\overline{\mu_{90}}$ shows the average upper limit from Feldman-Cousins method[25]. The upper limit is in unit of $\mathrm{eV} \cdot \mathrm{cm}^{-2} \cdot \mathrm{s}^{-1} \cdot \mathrm{sr}^{-1}$.

$\left.c m^{-2} \cdot s^{-1} \cdot s r^{-1}\right)$ can be set with $90 \%$ C.L. by the CRTNT experiment. It falls to $6.7\left(\mathrm{eV} \cdot \mathrm{cm}^{-2} \cdot \mathrm{s}^{-1} \cdot \mathrm{sr} \mathrm{r}^{-1}\right)$ for three years observation. Figure8 shows the corresponding results comparing with other experiments. As references, three neutrino source models together with atmospheric and cosmogenic neutrino models are plotted in the same figure as well.

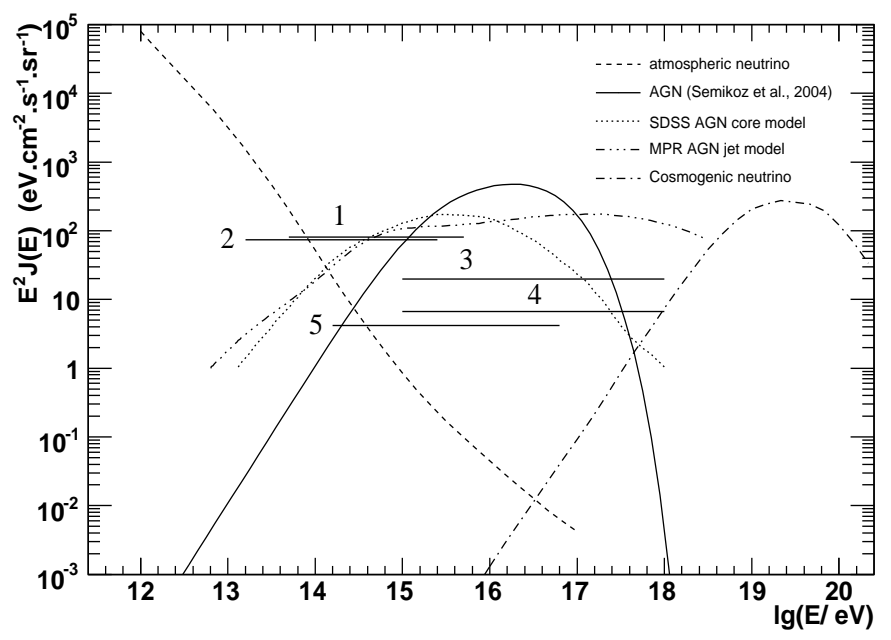

Figure 8. Predicted diffuse neutrino fluxes and sensitivities of CRTNT project (horizontal solid lines) for tau neutrinos and other three experiments for muon neutrinos. The lines marked with numbers represent the upper limit of different experiments. Line 3 and Line 4 are for CRTNT with one year and three year of observation, respectively. Line 1 is for Antares in one year of observation[?]. Line 2 is for Amanda II within 807 days observation 22. Line 5 is for IceCube with three year of observation 27.

\section{Conclusions}

A complete simulation chain is developed including neutrino interaction, $\tau$ lepton decay, air shower and light production, detector responses, triggering, and neutrino 
event selection algorithm. The event rate for a proposed AGN neutrino flux (Semikoz et. al, 2004) is found to be 28.6 per year. The event rates for MPR AGN jet model and SDSS AGN core model are 21.9 and 4.7, respectively.

Comparing with our previous estimate for the Mt. Wheeler site [7, the expected annual event rate increases from 8 for three sites at Mt. Wheeler Peak to 28.6 for four sites at Mt. Balikun site according to the same AGN model. The significant improvement comes from 1) a much larger FOV of the CRTNT detector array at the Mt. Balikun site because the mountain stretches longer with a height about $4 \mathrm{~km}$ a.s.l.; 2) an improvement of the detector design by widening the bandwidth of light signals which are composed mainly of scattered Cherenkov light distributing over a range up to $600 \mathrm{~nm}$; and 3) a correction of an error in the previous simulation code about Rayleigh scattering of Cherenkov photons which caused an underestimation of shower trigger efficiency by a factor of 2.5. The overall effect is about an enhancement of the event rate by a factor of 4.5. Other improvements have been made in the simulation by using more detailed description about neutrino interaction, $\tau$ lepton propagation in the mountain and its decay. Air shower generation is also improved as well.

According to a parametrization of $\mathrm{CR}$ event distribution, isotropic cosmic rays yield a background of 38300.7 events per year in a zenith angle ranging from $70^{\circ}$ to $75^{\circ}$. By using the neutrino event selection algorithm, $80.1 \%$ of 35.7 neutrino events with $0.4 \mathrm{CR}$ background event per year are picked out. If CRTNT does not see any signal excess with one year of observation, an upper limit of $19.9\left(\mathrm{eV} \cdot \mathrm{cm}^{-2} \cdot \mathrm{s}^{-1} \cdot \mathrm{sr}^{-1}\right)$ with $90 \%$ C.L. can be concluded.

\section{Acknowledgments}

This work and CRTNT project is supported by the Innovation fund (U-526) of the Institute of High Energy Physics (IHEP) and Hundred Talents \& Outstanding Young Scientists Abroad Program (U-610/112901560333) from IHEP and Chinese Academy

of Sciences. Authors MAH and TCL are supported by National Science Council of Taiwan under project number NSC-95-2112-M239-003 and NSC-96-2112-M239-001.

\section{References}

[1] J. G. Learned and K. Mannheim, 2000, Ann. Rev. Nucl. Part. Sci. 50679.

[2] E. Waxman, 2003, Nucl. Phys. B (proc. Suppl)118 353.

[3] M. Walter for the IceCube collaboration, 2007, Nucl. Phys. B (proc. suppl.) 172 13-16.

[4] G. Anton for the Antares collabaration, 2005, Nucl. Phys. B (proc. suppl.) 143 351-354.

[5] R. U. Abbasi et al., (HiRes collabaretion), 2008, astro-ph0803.0554

[6] C. Aramo, et al., (Auger collabaretion), 2005, Astropart. Phys. 23 65-77.

[7] Z. Cao, M. A. Huang, P. Sokolsky, 2005, J. Phys. G: Nucl. Part. Phys. 31 571-582.

[8] G. Aielli et al., (ARGO collabaretion), 2006, Nucl. Instr. and Meth. A 562, 92.

[9] D. Semikoz, G. Sigl, 2004, J. Cosmol. Astropart. Phys. 04(3) 1475-7516

[10] K. Mannheim, R. J. Protheroe and J. P. Rachen, 2000, Phys. Rev. D 63023003.

[11] F. W. Stecker, C. Done, M. H. Salamon and P. Sommers, 1992, Phys. Rev. Lett. 692738.

[12] F. W. Stecker, 2005, Phys. Rev. D 72107301.

[13] J. Pumplin et al., 2002, JHEP 0207012.

[14] G. Ingelman, A. Edin, J. Rathsman, 1997, Comput. Phys. Commun. 101 108-134: LEPTO web page: http://www3.tsl.uu.se/thep/lepto

[15] M. A. Huang, 2008, Nucl. Phys. B (proc. suppl.) 175-176 472-475

[16] R. Decker et al., 1993, Comp. Phys. Commun. 76361 ; (1992) 70 69; (1990) 64275 ; Updates of TAUOLA: P. Golonka et al., CERN-TH 2003-287, hep-ph/0312240 
[17] D. Heck, J. Knapp, J. N. Capdevielle, et al., 1998, FZKA-6019 Institute fur Kernphys. University of Karlsruhe

[18] A. J. Krueger, R. A. Minzner, 1976, Journal of Geophysical Research. 81 4477-4481.

[19] K. Kamata, J. Nishimura, 1958, Prog. Theoretical Phys. Suppl. 6 93-100.

[20] D. R. Longtin, A wind dependent desert aerosol model: radiative properties, Air Force Geophysics Laboratory, AFL-TR-88-0112, 1998.

[21] T. Abu-Zayyad, K. Belov, D. J. Bird, et al., 2001, Astropart. Phys. 16 1-11.

[22] A. Achterberg et al., (AMANDA collaboration), 2007, Phys. Rev. D 76042008.

[23] T. Abu-Zayyad, K. Belov, D. J. Bird, 2001, Astrophys. J. 557 686-699.

[24] G. C. Hill, K. Rawlins, 2003, Astropart. Phys. 19 393-402.

[25] G. J. Feldman, D. Robert. Cousins, 1998, Phys. Rev. D 57 3873-3889.

[26] T. Montaruli et al., (Antares collaboration), 2005, Acta Phys. Polon. B 36 509-518; hepex/0410079.

[27] J. Ahrens et al., (IceCube collaboration), 2004, Astropart. Phys. 20 507-532. 